Pierre-Alain Duc, Jonathan Braine and Elias Brinks, eds.

\title{
Head-on collisions: how to bring large quantities of gas out of inner disks
}

\author{
Jonathan Braine \\ Observatoire de Bordeaux, UMR 5804, CNRS/INSU, B.P. 89, F-33270 \\ Floirac \\ U. Lisenfeld \\ Instituto de Astrofísica de Andalucía, CSIC, Apdo. Correos 3004, 18080 \\ Granada, Spain \\ P.-A. Duc \\ CNRS URA 2052 and CEA/DSM/DAPNIA, Service d'Astrophysique, \\ Saclay, 91191 Gif sur Yvette cedex, France
}

\begin{abstract}
Head-on collisions of spiral galaxies can bring large quantities of gas out of spiral disks and into the intergalactic medium. Only two clear cases (UGC 12914/5 and UGC 813/6) of such collisions are known (Condon et al. 1993, 2002) and in both cases several $10^{9} \mathrm{M}_{\odot}$ of neutral gas is found in the bridge between the two galaxies which are now separating. About half of the gas is molecular. The gas, atomic or molecular, is brought out by collisions between clouds, which then acquire an intermediate velocity and end up between the galaxies. The bridges contain no old stars and in each case only one HII region despite the large masses of molecular gas, such that the star formation efficiency is very low in the bridges. The collisions occurred $20-50$ million years ago, much greater than the collapse time for dense cores. We (Braine et al. 2003,2004 ) show that collisions between molecular clouds, and not only between atomic gas clouds, bring gas into the bridges. It is not currently known whether the galaxies and bridges are bound or whether they will continue to separate, releasing several $10^{9} \mathrm{M}_{\odot}$ of neutral gas into the intergalactic medium.
\end{abstract}

During cloud collisions, the gas is ionized but then cools very quickly and becomes denser, allowing rapid recombination and $\mathrm{H}_{2}$ formation (e.g. Harwit et al. 1987). The denser the clouds initially, the quicker the gas becomes neutral and then molecular. The molecular gas observed in the bridges actually left the colliding disks having already recombined into $\mathrm{HI}$ and $\mathrm{H}_{2}-$ this did not take place in the bridge. To explain the low level of star formation in the bridges, we speculate that the dense pre-stellar cores were able to expand after ionization and lose their pre-stellar nature, delaying the onset of star formation in the bridges. The velocities of the collisions discussd here are close to the relative velocities of encounters in clusters. A simple calculation reveals that collisions between the neutral interstellar media (ISMs) of galaxies in dense environments 
could be an efficient mechanism for bringing gas out of the inner parts of spiral galaxies and into the intracluster medium.

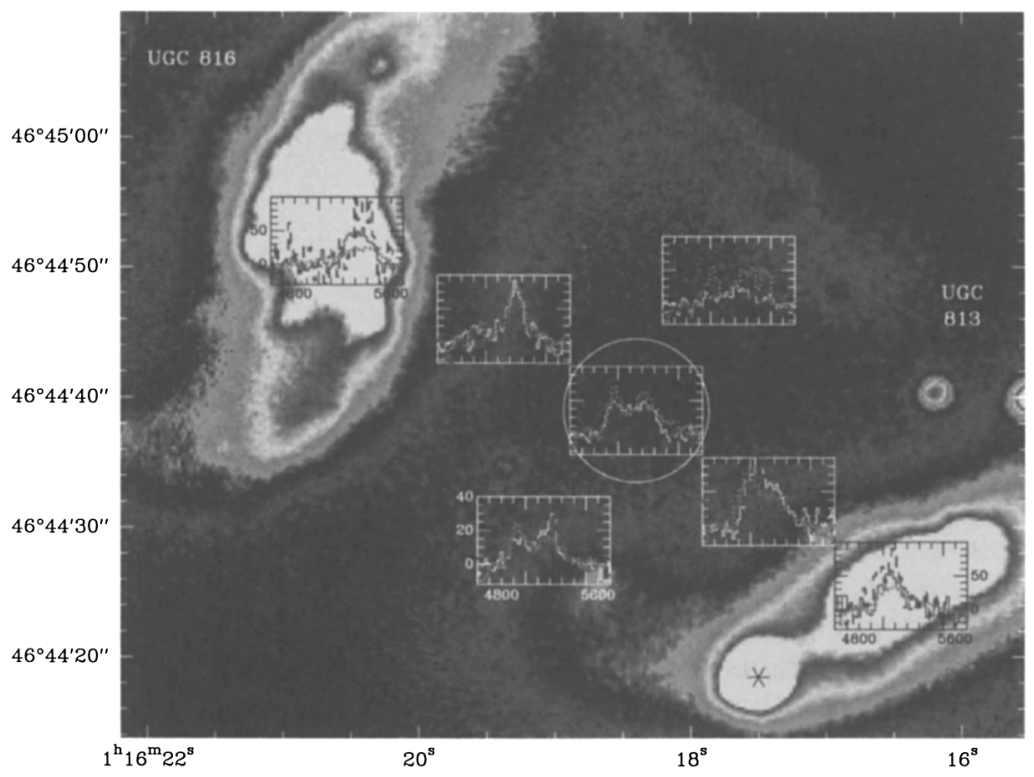

Figure 1. From Braine et al. 2004, $\mathrm{CO}(1 \rightarrow 0), \mathrm{CO}(2 \rightarrow 1)$, and $\mathrm{HI}$ spectra at the positions observed in the UGC 813/6 galaxies and bridge, using respectively solid, dashed, and dotted lines. UGC 813 is the edgeon spiral to the lower right and UGC 816 is to the left, less inclined and at a higher velocity. The asterisk marks a bright foreground star. For the CO spectra, the intensity scale is main beam antenna temperature in $\mathrm{mK}$ (-30 to $100 \mathrm{mK}$ for the galaxy centers and -12 to $40 \mathrm{mK}$ for the bridge positions). The Hi spectra are plotted such that equal $\mathrm{CO}(1 \rightarrow 0)$ and $\mathrm{HI}$ intensity correspond to equal $\mathrm{H}$-atom column densities in $\mathrm{H}_{2}$ and in $\mathrm{HI}$. It is thus apparent that in the bridge, the $\mathrm{HI}$ and $\mathrm{H}_{2}$ column densities are similar, assuming the $N\left(\mathrm{H}_{2}\right) / I_{\mathrm{CO}(1-0)}$ factor is correct. The underlying image is an $\mathrm{R}$ band image taken by Simon Verley with the IAA 1.5-m telescope.

\section{References}

Braine J., Davoust E., Zhu M., et al. 2003, A\&A 408 L13

Braine J., Lisenfeld U., Duc P.-A., et al 2004 A\&A in press

Condon J. J., Helou G., Sanders D. B., Soifer B. T. 1993, AJ 105, 1730

Condon J. J., Helou G., Jarrett T. H 2002, AJ 123, 1881

Harwit M., Houck J. R., Soifer B. T., Palumbo, G. G. C. 1987, ApJ 315, 28 